\title{
EL IMPACTO DE LA COVID EN LA DISTRIBUCION DE COMPETENCIAS
}

\author{
Paloma Biglino Campos \\ Catedrática de Derecho Constitucional, \\ Universidad de Valladolid
}

Cómo citar este artículo / Citation: Biglino Campos, P. (2021). El impacto de la covid en la distribución de competencias. Tudela Aranda, J. (coord.)

Estado Autonómico y covid-19,

Colección Obras colectivas, Fundación Manuel Giménez Abad, Zaragoza.

DOI: https://doi.org/10.47919/FMGA.OC21.0201

SUMARIO: I. INTRODUCCIÓN - II. LA COMPLEJIDAD DEL MARCO COMPETENCIAL - 1. Las normas que distribuyen competencias - 2. Las normas que influyen sobre la distribución de competencias - III. LA FLEXIBILIDAD DEL EJERCICIO DE LAS COMPETENCIAS - 1. La fase de centralización - 2. La fase de coordinación - 3. La fase de cogobernanza - IV. UN TEMA PENDIENTE: LAS GARANTÍAS DEL SISTEMA AUTONÓMICO - 1. Las garantías políticas -2 . Las garantías jurídicas

\section{INTRODUCCIÓN}

Ha pasado casi un año desde que se dieron en España los primeros casos de covid-19 y en el momento de escribir estas páginas seguimos bajo los efectos de la pandemia. En marzo de 2019, cuando estaba ya claro que no era posible detener la enfermedad sin tomar medidas de naturaleza excepcional, se pensaba que la situación podía superarse en pocas semanas. Ahora todos sabemos que no ha sido así, sino que las restricciones a la movilidad, el uso obligatorio de mascarillas, los cierres comerciales, entre otras acciones 
adoptadas para frenar los índices de contagios y de fallecidos, se han prolongado en el tiempo, si bien su intensidad ha ido variando según la crudeza de las oleadas.

La extensión temporal de la lucha contra la pandemia, así como las oscilaciones en las políticas ideadas para hacerle frente complica mucho el análisis y obliga a reconocer ciertas limitaciones.

La primera de ellas parte de admitir que las medidas adoptadas para luchar contra la covid, tanto por el Estado como por las Comunidades Autónomas, distan de haber alcanzado el consenso que hubiera sido deseable. A veces, se han dejado de lado criterios meramente científicos para hacer batalla política sobre la adopción de terminadas decisiones. No resulta fácil entender por qué la colaboración de las farmacias en la realización de los test de anticuerpos se considera una propuesta de derechas, mientras que las precauciones frente a ella se tildan de progresistas. A la hora de escribir sobre el reparto de competencias, resulta difícil abstraerse de este tipo de polémicas. Más difícil todavía es evitar que cualquier reflexión sobre el asunto no sea leída en términos meramente políticos, con la finalidad de adscribirla a una u otra ideología.

La segunda de las limitaciones afecta al objeto de estas páginas y consiste en reconocer que, hasta el momento, no se ha podido demostrar que la estructura territorial o la distribución de competencias que corresponde a cada forma de Estado haya sido determinante del éxito o el fracaso en la lucha contra la pandemia. En efecto, los resultados no han sido muy distintos en Estados unitarios, como es Francia, y en Estados federales, como es la República Federal de Alemania'.

La influyente revista The Lancet publicaba hace pocos meses $^{2}$ los resultados de una investigación en la que se comparaban las distintas formas de hacer

\footnotetext{
${ }^{1}$ Carbajosa, A., Bassets, M., "La pandemia examina el federalismo alemán y el centralismo francés", El País, 18 abril 2020. Disponible en: https://elpais.com/internacional/2020-04-17/lapandemia-examina-el-federalismo-aleman-y-el-centralismo-frances.html,

${ }^{2}$ Han, E., Mei Jin Tan, M., Turk, E., Sridhar, D., Leung, G. M., Shibuya, K. et alii, "Lessons learnt from easing COVID-19 restrictions: an analysis of countries and regions in Asia Pacific and Europe", The Lancet, 24 septiembre 2020, Disponible en: https://www.thelancet.com/pdfs/journals/lancet/PIIS0140-6736(20)32007-9.pdf.
} 
frente a la enfermedad en varios países de Asia y Europa. Concluía afirmando que las estrategias que habían generado más éxito eran las que, entre otros extremos, se basaban en los siguientes factores: un buen conocimiento de la expansión de la infección, obtenido mediante estadísticas fiables; el compromiso de los ciudadanos en la lucha contra la pandemia, que se consigue más fácilmente cuando los criterios establecidos por los poderes públicos son claros y generales; una acción decidida de la sanidad pública para testar, trazar y aislar a los contagiados; un alto grado de disponibilidad de tratamientos, equipamientos (unidades de cuidados intensivos, ventiladores) y personal médico para hacer frente a la enfermedad, así como una acción decidida en el control de fronteras.

Es evidente que la respuesta que ha dado España a algunas de estas exigencias ha estado condicionada por nuestra estructura territorial. Ahora bien, también hay que reconocer que han influido otros factores en los que el Estado de las Autonomías tiene mucho menor peso, como son, por ejemplo, los recortes que la crisis económica impuso al sistema sanitario o la incidencia que el sector turístico tiene en nuestra economía.

La tercera de las limitaciones tiene un carácter metodológico. A día de hoy, el Código sobre covid-19 publicado por el BOE contiene 341 normas sólo de Derecho estatal. Para conocer a fondo la respuesta de nuestro ordenamiento a la enfermedad habría que añadir, además, las disposiciones adoptadas por las Comunidades Autónomas, que son muy abundantes. Sirva como ejemplo que una búsqueda en el portal de normativa vasca Legegunea genera al menos 92 resultados. A esto sería preciso sumar las numerosas resoluciones adoptadas por la jurisdicción contencioso administrativa sobre el asunto. Baste con citar que, a 5 de abril de 2021, la página web del Centro de Documentación Judicial (CENDOJ) arroja 6.140 resultados sobre el Real Decreto de alarma sanitaria covid-19.

Esta complejidad obliga a delimitar el objeto de estudio y seleccionar sólo los aspectos que pueden ser más significativos para entender qué ha sucedido con la distribución de competencias. Siguiendo esta orientación, las próximas páginas se centrarán en un asunto que, por su incidencia en los derechos fundamentales, ha sido especialmente polémico. Me refiero a la debatida actuación de los poderes públicos a la hora de imponer confinamientos, sean 
estos perimetrales o toques de queda nocturnos. Será preciso, por tanto, dejar de lado otros temas que, sin duda, son de interés, como son el reparto territorial de poder que ha afectado al ejercicio del derecho de reunión, las relaciones exteriores, el derecho a la educación o la libertad de empresa. Ahora bien, como se pondrá de manifiesto más adelante, estos otros asuntos suscitan menos problemas competenciales. Frente a ello, las medidas que han limitado la libertad de movimiento no sólo han sido las más gravosas, sino también las más polémicas. Sirva como ejemplo que los confinamientos domiciliarios del primer estado de alarma fueron de tanta intensidad que cabe preguntarse, al menos como hipótesis, si no han llegado a afectar a la libertad personal reconocida en el artículo $17 \mathrm{CE}^{3}$. Esas y otras restricciones a la libre circulación han generado, además, tensiones territoriales desconocidas hasta ahora. La diversidad de criterios entre el Estado y Madrid sobre los confinamientos perimetrales generaron el primer enfrentamiento grave entre la entidad central y una Comunidad Autónoma que carece de hecho diferencial. Enfrentamiento que, como todos sabemos, se saldó con la declaración del estado de alarma sólo para dicha Comunidad.

La complejidad de la situación y la abundancia de datos normativos aconsejan, además, renunciar a hacer un relato cronológico de todo lo que ha pasado desde el primer estado de alarma. Esta tarea recopilatoria es, sin duda, importante, pero excedería con mucho del número de páginas que tengo asignado. Por esta razón, es conveniente centrarse en exponer las conclusiones a las que conduce el análisis de lo que ha ido sucediendo durante todo este periodo. Así, en el siguiente apartado se analizará el complejo marco competencial que afecta a la gestión de la pandemia, que no sólo está integrado por el artículo 149.1 y los Estatutos de Autonomía, sino por otros muchos preceptos constitucionales y legales. Posteriormente se estudiará la desigual manera en que dicha distribución de poder se ha llevado a la práctica. El trabajo se cierra con una reflexión acerca de cómo habría sido posible gestionar de manera más eficaz la lucha contra la pandemia desde el punto de vista territorial, activando las garantías políticas y jurídicas de nuestro Estado de las autonomías.

\footnotetext{
${ }^{3}$ Durán Alba, J. F., "Afectaciones a la libertad de circulación derivadas del estado de alarma" en Biglino Campos, P.; Durán Alba, F. Los efectos horizontales de la covid-19 sobre el ordenamiento constitucional: estudios sobre la primera oleada, Fundación Manuel Giménez Abad, Zaragoza, 2020 DOI: https://doi.org/10.47919/FMGA.OC20.0010
} 


\section{LA COMPLEJIDAD DEL MARCO COMPETENCIAL}

En otro lugar he tenido ocasión de referirme a los efectos horizontales que la covid-19 ha tenido sobre nuestro sistema constitucional ${ }^{4}$. En ese momento advertía que, desde la entrada en vigor de la Constitución, ningún otro acontecimiento ha tenido un impacto equiparable tanto sobre la parte dogmática como sobre la parte orgánica de nuestra norma fundamental. No sólo han sufrido los derechos que concretan los principios de igualdad y libertad, sino que también las relaciones gobierno y parlamento han resultado afectadas. No es de extrañar, pues, que la organización territorial del Estado también haya resultado alterada. El primer dato a destacar en este ámbito es la complejidad del marco normativo porque, en la acción contra la enfermedad, no sólo han estado implicadas normas que regulan directamente la distribución de competencias sino también otras que, aunque con un contenido distinto, han afectado a la distribución de poder.

\section{Las normas que distribuyen competencias}

Es claro que el primer precepto a tener en cuenta es el artículo 149.1.16 de la Constitución. Esta norma reserva al Estado exclusivamente las bases y la coordinación general de la sanidad, por lo que todas las Comunidades han asumido competencias de legislación de desarrollo y ejecución sobre la misma materia ${ }^{5}$. Hay que tener presente, también, que la acción contra la pandemia puede encuadrarse en otros títulos competenciales, como son las que la Constitución atribuye al Estado a la hora de velar por la igualdad de todos los españoles en el ejercicio de los derechos y en el cumplimiento de los deberes constitucionales y a la hora de dictar las bases y la coordinación de la planificación general de la actividad económica (art. 149.1.1 y 13).

\footnotetext{
4 Biglino Campos, P., "Introducción", Biglino Campos, P.; Durán Alba, F. Los efectos horizontales de la COVID sobre el sistema constitucional: estudios sobre la primera oleada, Fundación Manuel Giménez Abad, Zaragoza, 2020. DOI: https://doi.org/10.47919/FMGA.OC20.0001

${ }^{5}$ Delgado del Rincón, L., "Algunas consideraciones sobre el Derecho a la protección de la salud y el bien jurídico de la salud colectiva en tiempos de pandemia" en Biglino Campos, P.; Durán Alba, F. Los efectos horizontales de la COVID sobre el sistema constitucional, Fundación Manuel Giménez Abad, Zaragoza, 2020. DOI: https://doi.org/10.47919/FMGA.OC20.0017
} 
Más adelante será preciso volver sobre la función de coordinación estatal. Por el momento, es preciso destacar que el Estado ha hecho uso de sus competencias mediante el dictado de un conjunto de leyes que son manifiestamente mejorables.

La primera de ellas tiene naturaleza orgánica y es la LO 3/1986, de 14 de abril, de Medidas Especiales en Materia de Salud Pública. Durante algún tiempo, esto es, entre el primer y el segundo estado de alarma, los confinamientos perimetrales se fundamentaron en el artículo 3 de dicha Ley. Dicho precepto autoriza a la autoridad sanitaria a adoptar "las medidas oportunas para el control de los enfermos, de las personas que estén o hayan estado en contacto con los mismos y del medio ambiente inmediato, así como las que se consideren necesarias en caso de riesgo de carácter transmisible". Para destacados sectores doctrinales ${ }^{6}$ así como para determinados Tribunales de Justicia $^{7}$, esta disposición no posee el grado de certeza y previsibilidad que, según la jurisprudencia del Tribunal Constitucional, la seguridad jurídica demanda cuando se trata de limitar derechos fundamentales. Ahora bien, esta polémica no afecta a la distribución de competencias, dado que la deficiencia, de existir, afectaría a los actos limitativos de derechos tanto del Estado como de las Comunidades Autónomas porque la Ley no sólo se refiere al primero sino, de manera mucho más genérica, a "las autoridades sanitarias competentes".

Hay que tener en cuenta, además, otras dos leyes estatales, esto es, la Ley 14/1986, General de Sanidad y la Ley 33/2011, General de Salud Pública. Lo más interesante de estas normas es que ambas tienen carácter básico y que ambas permiten a las autoridades sanitarias, tanto del Estado como de las Comunidades Autónomas, cuando existe un riesgo inminente y extraordinario para la salud, adoptar medidas tales como la suspensión del ejercicio de actividades, el cierre preventivo de instalaciones, establecimientos, servicios e

\footnotetext{
6 Sáenz Royo, E., Respuesta a Doménech, G. "Comunidades Autónomas, derechos fundamentales y covid-19", Almacén de Derecho, 21 julio 2020. Disponible en: https://almacendederecho.org/comunidades-autonomas-derechos-fundamentales-y-covid-19; De la Quadra-Salcedo Janini, T., "Estado autonómico y lucha contra la pandemia" en Biglino Campos, P.; Durán Alba, Los efectos horizontales de la COVID sobre el sistema constitucional Fundación Manuel Giménez Abad, Zaragoza, 2020, DOI: https://doi.org/10.47919/FMGA.OC20.0005

${ }_{7}$ Por ejemplo, el Auto 332/2020, de 10 de octubre, de la Sección primera de la Sala de lo Contencioso Administrativo del Tribunal Superior de Justicia de Aragón.
} 
industrias, así como otras medias que se estimen sanitariamente justificadas. Esto significa que los cierres de bares y restaurantes, establecimientos comerciales, gimnasios, etc., decididos por las Comunidades Autónomas antes del segundo estado de alarma no plantean tantos problemas competenciales, sobre todo cuando han sido acordados como consecuencia de una habilitación estatal, como es el caso. Por último, cabe citar la Ley 16/2003, de 28 de mayo, de cohesión y calidad del Sistema nacional de Salud, a la que más adelante deberé hacer referencia.

\section{Las normas que influyen sobre la distribución de competencias}

Ahora bien, como se señalaba antes, además de las leyes de sanidad, también es preciso tener en cuenta otras disposiciones que, sin ser competenciales, influyen sobre el reparto de poder. La primera de ellas es la LO 4/1981 que, en desarrollo del artículo $116 \mathrm{CE}$, regula los estados de alarma, excepción y sitio. Desde el punto de vista de la organización territorial del poder, el estado de alarma permite una centralización de las competencias, aunque no obliga a ella, dado que su artículo 7 permite al Gobierno delegar sus funciones en el Presidente de la Comunidad Autónoma cuando la declaración afecte sólo al territorio de una de ellas. Sobre este extremo será preciso volver en el siguiente apartado.

Por último, es preciso tener en cuenta el artículo 81.1 de la Constitución, que reserva a la ley orgánica, esto es, al Parlamento nacional, la regulación de los derechos fundamentales. Ahora bien, esta capacidad normativa del Estado no le atribuye una competencia exclusiva en materia de derechos fundamentales. En efecto, los derechos fundamentales no son competencias, sino que tienen un efecto horizontal, porque son límites y fuente de obligaciones para todos los poderes públicos. Esto significa que, al margen de la regulación que le corresponde al Estado, no sólo esta entidad, sino también las Comunidades Autónomas están obligadas a respetarlos y a implementarlos en el marco de sus competencias.

Es cierto que las medidas adoptadas por dichas estructuras territoriales para hacer frente a la pandemia han provocado conflictos de derechos fundamentales, ya que restringieron la libertad de movimiento para salvaguardar el derecho a la salud. Este, como reconoce la jurisprudencia del 
Tribunal Constitucional, enlaza con otro derecho fundamental, esto es, el derecho a la vida y a la integridad física ${ }^{8}$. También es verdad que este conflicto debe ser resuelto ponderando, en cada caso, los dos derechos conforme al principio de proporcionalidad. Ahora bien, este juicio afecta a los aspectos sustantivos de las normas, no a sus aspectos formales. Nada nos dice, pues, sobre la autoridad que puede adoptar la medida. Este es otro problema, que tiene que resolverse en función de los títulos competenciales. $Y$, en este aspecto, no conviene olvidar que la gestión de todo el aparato establecido para salvaguardar la salud corresponde a las Comunidades Autónomas.

Junto a las disposiciones analizadas, hay que tener en cuenta que existen otras previsiones que también pueden afectar a la distribución de competencias, limitando la actuación de los poderes públicos. En efecto, sería necesario preguntarse por la incidencia que puede tener el artículo 139.2 CE sobre algunos de los confinamientos territoriales decididos por las Comunidades Autónomas, previa delegación del Estado mediante el artículo 6 del RD 926/2020. Recordemos que, según dicho precepto constitucional, ninguna autoridad puede adoptar medidas que directa o indirectamente obstaculicen la libertad de circulación de las personas en todo el territorio español.

El marco competencial es, como vemos, decididamente complejo. Ahora bien, no creo que sea posible concluir que los problemas y las polémicas que se han suscitado en estos meses acerca de la titularidad del poder para limitar la libertad de movimiento tengan su origen en esta pluralidad de disposiciones. En efecto, cualquier sistema de distribución de competencias suscita problemas porque la realidad se resiste a ser dividida en compartimentos estancos. En todo sistema jurídico siempre aparecerán dudas y visiones encontradas acerca de la titularidad de las atribuciones ya que, sobre la misma materia, inevitablemente se entrecruzan títulos competenciales distintos. Las dificultades se acrecientan cuando, como sucede en casi todos los federalismos excepto el estadounidense, el modelo no es dual, sino que las funciones se reparten entre entidades territoriales diferentes.

No parece, pues, que el problema tenga fácil solución. El federalismo tiene indudables ventajas, como son aproximar la decisión a los ciudadanos, garantizar el pluralismo político y dar expresión a los hechos identitarios. Ahora

\footnotetext{
${ }^{8}$ STC $160 / 2007$, entre otras.
} 
bien, también plantea algunos inconvenientes, como es el solapamiento y el entrecruzamiento de las competencias. Es cierto que una reforma de la Constitución podría afrontar alguna de estas dificultades. Pero también es verdad que cualquier fórmula que se implantara generaría los mismos o parecidos problemas.

\section{LA FLEXIBILIDAD DEL EJERCICIO DE LAS COMPETENCIAS}

El principal hecho que dificulta analizar la manera en que se ha puesto en marcha el marco competencial que se acaba de mencionar radica en que, a lo largo de la pandemia, no siempre se ha interpretado de la misma manera. En efecto, el Estado ha ejercido sus propias atribuciones siguiendo tres orientaciones distintas que, a veces, se han simultaneado. Como se analizará a continuación, tras una primera etapa de centralización de las competencias, y con el objetivo de superar los reproches que había recibido por parte de algunas Comunidades Autónomas, el Estado opto por ejercer su función de coordinación para, posteriormente, pasar a una fase de cogobernanza.

\section{La fase de centralización}

Como se acaba de señalar, el Real Decreto 463/2020, de 14 de marzo, por el que se declara por primera vez el estado de alarma para la gestión de la situación de crisis sanitaria ocasionada por el covid-19, generó una clara centralización de las competencias. En esta primera fase, la autoridad competente pasó a ser el Gobierno (art. 4) especialmente en materia de sanidad. En consecuencia, el Ejecutivo nacional se reservó la adopción de las decisiones principales, mientras que las Administraciones de las Comunidades Autónomas conservaron las competencias para la gestión ordinaria de sus servicios, siempre en el marco de las órdenes dictadas por la autoridad competente (art. 6). Este Real Decreto fue, además, el que provocó mayores limitaciones de la libertad de movimiento, dado que sólo se autorizaba la circulación de las personas para las actividades previstas en el artículo 7 de dicha norma.

La nueva normalidad, de la que se tratará más adelante, no supuso en todos los casos la devolución del ejercicio de competencia a las Comunidades 
Autónomas. En efecto, algunos meses más tarde, el RD 900/2020, de 9 de octubre, mantuvo la tendencia centralizadora. Dicha norma sólo declaraba el estado de alarma en determinados municipios de la Comunidad Autónoma de Madrid (entre ellos la capital) y volvía a atribuir al Gobierno la calidad de autoridad competente (art. 4). En su virtud se restringía la entrada y salida de las personas de las zonas afectadas, mientras que el artículo 8, al regular la gestión de los servicios, reconocía que cada Administración conservaba las competencias establecidas en la legislación vigente.

\section{La fase de coordinación}

Después de esta primera fase, y en paralelo a la actuación con respecto sólo a Madrid, la intervención del Estado siguió otra orientación. La centralización que había producido el primer estado de alarma suscitó las críticas de parte de la oposición y de algunas Comunidades Autónomas ${ }^{9}$. Por este motivo, el Estado siguió ejerciendo sus competencias, pero, esta vez, mediante la función de coordinación que recoge el artículo 149.1.16 de la Constitución.

Antes de analizar lo que ocurrió en esta segunda etapa, hay que reconocer las dificultades que conlleva identificar la naturaleza de dicha función. En efecto, la jurisprudencia constitucional sobre el asunto dista de estar clara. Aun así, se pueden destacar dos notas. En primer lugar, "que atribuye al Estado cierto poder de dirección", ya que corresponde a dicha entidad "la fijación de medios y de sistemas de relación que hagan posible la información recíproca, la homogeneidad técnica en determinados aspectos y la acción conjunta de las autoridades estatales y autonómicas en el ejercicio de sus respectivas competencias". Y, en segundo lugar, que "presupone lógicamente que hay algo que debe ser coordinado; esto es, la existencia de competencias de las Comunidades Autónomas en materia de sanidad, que el Estado, al coordinarlas, debe obviamente respetar"10 (ver, por todas, la STC 140/2017, f.j. 3).

\footnotetext{
${ }^{9}$ Romero, J., "Sánchez da a las CCAA la gestión de la fase 3 para amarrar la prórroga con ERC y PNV", El Confidencial, 30 mayo 2020, Disponible en: https://www.elconfidencial.com/espana/2020-05-30/pedro-sanchez-ccaa-gestion-fasedesescalada-pnv-erc 2617955/

${ }^{10}$ Ver, por todas, la STC 140/2017, f. j. 3.
} 
La actuación coordinada del Estado, que comenzó a principios del verano de 2020, está regulada en el artículo 65 de la Ley 16/2003, de cohesión y calidad del Sistema Nacional de Salud. Según este precepto, la declaración de actuaciones coordinadas corresponde al Ministro de Sanidad, previo acuerdo del Consejo Interterritorial del Sistema Nacional de Salud. Según el artículo 151 de la Ley 40/2015, los acuerdos que se adopten son de obligado cumplimiento para todas las Administraciones Públicas integrantes del Consejo, con independencia del sentido de su voto.

No voy a describir las veces que el Consejo Interterritorial se ha reunido ni los acuerdos que ha adoptado. Para el tema que tratamos en estas páginas, el más polémico de todos fue el acuerdo de 30 de septiembre en virtud del cual era posible que las Comunidades Autónomas confinaran perimetralmente términos municipales ${ }^{11}$. También revistió interés el acuerdo de 22 de octubre, aunque este no afectó a la libertad de movimiento ${ }^{12}$. En el mismo, por primera vez se fijaron indicadores para establecer cuatro niveles de riesgo y las medidas a adoptar en cada uno de ellos. Ahora bien, se trataba de meras recomendaciones no obligatorias para las Comunidades Autónomas, asunto que se tratará más adelante.

La actuación coordinada, tal y como se ha llevado a cabo en nuestro país, ha encontrado obstáculos. Ya se ha mencionado que, en algunas ocasiones, no ha tenido carácter vinculante. Pero, además, cuando ha tenido dicha fuerza, ha suscitado reacciones dispares por parte de los Tribunales ordinarios de Justicia.

Los primeros problemas se remontan a principios de verano, cuando Gobiernos de distintas Comunidades Autónomas empezaron a confinar a grupos concretos de población y limitar algunos derechos, como el de asociación. Así sucedió, durante el mes de julio, en El Segría, en Lérida, en ciertas comarcas

\footnotetext{
${ }^{11}$ Resolución de 30 de septiembre de 2020, de la Secretaría de Estado de Sanidad, por la que se da publicidad al Acuerdo del Consejo interterritorial del Sistema Nacional de Saludo sobre la Declaración de Actuaciones Coordinadas en Salud Pública para responder ante situaciones de especial riesgo por transmisión no controlada de infecciones causadas por el SARS-Cov-2, de fecha 30 de septiembre de 2020. BOE 1 octubre 2020.

${ }^{12}$ Disponible en: en https://www.lamoncloa.gob.es/serviciosdeprensa/notasprensa/sanidad14/Documents/2020/221 020_ActuacionesrespuestaCOVID.pdf.
} 
agrícolas de Huesca y La Marina, de Lugo ${ }^{13}$. Posteriormente, al amparo de la resolución del Ministro de Sanidad por la que se declaraban las actuaciones coordinadas decididas por el Consejo Interterritorial el 30 de septiembre, se confinaron grupos de población cada vez más amplios.

Estas medidas fueron impugnadas primero, ante los Jueces de lo Contencioso Administrativo y, tras la reforma introducida por la Ley $3 / 2020^{14}$, ante las Salas de lo Contencioso-administrativo de los Tribunales Superiores de Justicia. Según la nueva redacción del artículo 10.8. la Ley 29/1998, reguladora de la Jurisdicción Contencioso-Administrativa, correspondía a dichos Tribunales la competencia para autorizar o ratificar las medidas adoptadas por la autoridad sanitaria que implicaran la limitación o restricción de derechos fundamentales cuando sus destinatarios no estuvieran identificados.

Las decisiones de la Jurisdicción Contencioso-Administrativa sobre el asunto fueron heterogéneas y divergían, especialmente, acerca de la interpretación del artículo 3 de la Ley Orgánica 3/1986, de Medidas Especiales en Materia de Salud Pública. En algunos casos, para no ratificar o autorizar las medidas, se utilizó el argumento al que se ha hecho referencia en el segundo apartado de este trabajo, ya que se consideró que dicho precepto era demasiado indeterminado para limitar la libertad de circulación u otros derechos fundamentales.

Entre estas decisiones, las que tuvieron quizá más impacto fueron el Auto 128/2020, de 8 de octubre de 2020, de la Sala de lo Contencioso Administrativo del Tribunal Superior de Justicia de Madrid, que no ratificó medidas sobre confinamientos perimetrales entre distintos municipios de la Comunidad Autónoma y el Auto 32/2020, de 22 de octubre de 2020, de la Sala de lo Contencioso-Administrativo del Tribunal Superior de Justicia del País Vasco. En esta ocasión, no se ratificaron las limitaciones al derecho de reunión decididas por la Consejera de Salud del Gobierno vasco.

\footnotetext{
13 "Nuevos confinamientos. Qué restricciones de movilidad puede haber durante las vacaciones por un rebrote". La Vanguardia, 6 julio 2020. Disponible en: https://www.lavanguardia.com/motor/actualidad/20200706/482149594995/restriccionesmovilidad-desplazamientos-vacaciones-confinamiento-brote-coronavirus-covid-19.html

${ }^{14}$ De 18 de septiembre, de medidas procesales y organizativas para hacer frente al COVID-19 en el ámbito de la Administración de Justicia.
} 


\section{La fase de cogobernanza}

Estas decisiones judiciales influyeron, seguramente, en la decisión del Gobierno de volver a declarar el estado de alarma, mediante el RD 926/2020, de 25 de octubre, que atribuía a las restricciones de derechos fundamentales un fundamento más sólido. Dicha norma inaugura una tercera etapa porque en esta ocasión, en vez de acudir a la centralización o al ejercicio de la competencia de coordinación, se siguió el modelo de cogobernanza.

Es cierto que artículo 2 del Real Decreto que se acaba de citar declara que la autoridad competente es el Gobierno de la Nación. Pero el mismo precepto recoge una delegación de dicha autoridad en los Presidentes las Comunidades Autónomas o ciudades con Estatuto de Autonomía. El Real Decreto, nuevamente, prevé restricciones a la libertad de circulación (como son los toques de queda nocturnos y los confinamientos perimetrales), al derecho de reunión (prohibición de reuniones de más de seis personas en lugares públicos o privados) y a la libertad de culto. Ahora bien, en virtud de lo dispuesto en el artículo 6, dichas medidas son acordadas por la autoridad competente delegada, previa comunicación al Ministerio de Sanidad. Son, pues, los Presidentes de las Comunidades Autónomas quienes pueden delimitar el horario del toque de queda, limitar la salida y entrada del territorio de cada Comunidad Autónoma o restringir la permanecía de personas en lugares de culto, dentro de los límites establecidos en el propio Real Decreto. Dichas autoridades pueden también modular, flexibilizar y hasta suspender dichas medidas con el alcance y ámbito territorial que determinen (art. 10).

Son muchos los interrogantes que suscita el Real Decreto del que venimos hablando. Sorprende, en primer lugar, que su exposición de motivos prevea una prórroga de seis meses, duración que fue confirmada por la autorización dictada por el Congreso de los Diputados el 29 de octubre de $2020^{15}$. Aunque ni el artículo 116.2 la Constitución ni el artículo 6 de la LO 4/1981 establecen expresamente un límite temporal a la duración de la prórroga, quizás haya límites implícitos a la misma. Hay que tener en cuenta, en primer lugar, que

\footnotetext{
${ }^{15}$ Resolución de 29 de octubre de 2020, del Congreso de los Diputados, por la que se ordena la publicación del Acuerdo de autorización de la prórroga del estado de alarma declarado por el Real Decreto 926/2020, de 25 de octubre, por el que se declara el estado de alarma para contener la propagación de infecciones causadas por el SARS-CoV-2. Disponible en: https://www.boe.es/buscar/act.php?id=BOE-A-2020-13492
} 
dichos preceptos establecen taxativamente que la duración del estado de alarma no puede superar los quince días, limitación que debería de tenerse en cuanta a la hora de fijar su extensión. Además, en segundo lugar, la restricción temporal puede deducirse de la propia excepcionalidad de la medida que, aunque no interrumpe el funcionamiento regular de las instituciones ni suspende los derechos fundamentales, altera de manera gravosa sus regímenes ordinarios.

También llama la atención la escasa rendición de cuentas prevista en el artículo 14 del RD 926/2020, limitada a la comparecencia quincenal del Ministro de Sanidad ante la Comisión de Sanidad y Consumo del Congreso de los Diputados (art. 14). El refuerzo del poder ejecutivo que conlleva el estado de alarma exigiría potenciar, y no debilitar, el control político que tanto el Congreso como el Senado ejercen sobre el Gobierno en cualquier democracia. Al menos, esta es la orientación a la que responde el artículo 116.6 CE, cuando afirma que la declaración de los estados de alarma, de excepción y de sitio no modificará el principio de responsabilidad del Gobierno y de sus agentes reconocidos en la Constitución y en las leyes.

Ahora bien, para el objeto de estas páginas, hay otros interrogantes que revisten todavía mayor importancia. El primero de ellos consiste en preguntarse si la solución adoptada es conforme a lo previsto en la propia LO 4/1981. Es verdad que el artículo 5 de esta norma establece que el estado de alarma sea declarado a petición del Presidente de una Comunidad Autónoma, mientras que el artículo 7 permite al Gobierno delegar en dicha autoridad. Pero esta solución está prevista para cuando los problemas que generan la declaración afecten exclusivamente a todo, o parte del ámbito territorial de una Comunidad Autónoma y la declaración afecte exclusivamente a todo o parte del territorio de una Comunidad.

La delegación en los Presidentes de todas las Comunidades autónomas no sólo parece ir en contra de dichas disposiciones, sino que, además, puede ser contraria a la concepción de las competencias que es característica de nuestro sistema jurídico. En España, no se trata de poderes que puedan ser ejercidos o dejados de ejercer por el Estado según el criterio político de la autoridad central. Es cierto que, en otros federalismos, como son la Unión Europea o la República Federal Alemana, una concepción más flexible de las competencias 
permite que la Federación se abstenga de actuar, aún en el marco de sus competencias exclusivas, en favor de los Estados miembros ${ }^{16}$. Ahora bien, no parece sencillo exportar esta flexibilidad a nuestro país, donde impera una concepción mucho más estricta de la competencia, ya que se interpreta como indeclinable.

Así, la competencia puede ser básica o de desarrollo, legislativa o ejecutiva, del Estado o de las Comunidades Autónomas. Pero, una vez determinada su titularidad, sólo podrá ejercerse por la entidad territorial habilitada para ello. Buena prueba de ello es que, en caso de que el Estado considere necesario transferir o delegar algunas de sus competencias, deberá acudir a las leyes marco de delegación de competencias legislativas previstas en el artículo 150.1 CE o a las leyes orgánicas de transferencia y delegación del artículo 150.2 CE. En este último caso se requiere, además, que se trate de competencias estatales que, por su naturaleza, sean susceptibles de transferencia 0 delegación. También hay que recordar que, en caso de que el Estado no ejerza su competencia, es posible activar los conflictos negativos de competencia previstos en el artículo 68 y siguientes de la LOTC ${ }^{17}$.

El modelo de cogobernanza plantea también problemas acerca de su eficacia. El problema no es la diversidad de las medidas adoptadas por las distintas Comunidades Autónomas. En efecto, esta variedad podría justificarse en razón de las distintas situaciones en las que se halla cada territorio y por la diversidad de criterios en la lucha contra la pandemia. Tampoco en Estados unitarios

\footnotetext{
${ }^{16}$ Recordemos que el artículo 2.1 del TFUE permite que la Unión habilite a los Estados para legislar y adoptar actos jurídicamente vinculantes en materias que corresponden a la Unión en régimen de exclusividad. Cuando la competencia es compartida, el artículo 2.2.1 permite que la Unión y los Estados miembros puedan legislar y adoptar actos jurídicamente vinculantes. Ahora bien, los Estados miembros ejercerán su competencia en la medida en que la Unión no haya ejercido la suya y ejercerán de nuevo su competencia en la medida en que la Unión haya decidido dejar de ejercer la suya.

Este régimen no es muy distinto al que existe en la Ley Fundamental de Bonn. Según su artículo 71, los Länder tienen la facultad de legislar en el ámbito de la legislación exclusiva de la Federación, siempre que una ley federal los autorice expresamente para ello. Pero, además, y según el artículo 72, en el ámbito de la legislación concurrente, los Länder tienen la facultad de legislar mientras y en la medida que la Federación no haya hecho uso mediante ley de su competencia legislativa. Además, el ap. 4 del mismo artículo autoriza a la Federación a determinar, mediante ley, que una regulación legislativa federal ya no sea necesaria, por lo que pueda ser reemplazada por la legislación de los Länder.

7 Biglino Campos, P., Federalismo de integración y de devolución: el debate sobre la competencia, Madrid, CEPC, 2007, pp. 170 y 208,
} 
como es Francia ${ }^{18}$ se ha tratado por igual las zonas rurales con pocos casos de contagio y las grandes urbes donde ese índice era elevado. El problema principal es que la diversidad no siempre se ha justificado en datos objetivos ni ha respondido a criterios comunes.

Es verdad que, como antes se señalaba, el Consejo interterritorial aprobó, el 22 de octubre de 2020, un documento de actuaciones de respuesta coordinada para el control de la transmisión de covid-19, en el que se fijan indicadores, niveles y medidas ${ }^{19}$. Ahora bien, dicho documento carece de valor vinculante para las Comunidades Autónomas. Han sido estas las que han tenido libertad para interpretarlo y aplicarlo en la forma en que lo han estimado conveniente. El mejor ejemplo de esta diversidad es lo que ha ocurrido en las navidades de 2020, donde se han dado 17 regímenes jurídicos distintos en lo que se refiere a movilidad con otras regiones, horarios del toque de queda o número de personas asistentes a comidas y cenas $^{20}$.

Un buen ejemplo de los problemas que estas distintas respuestas pueden generar es lo sucedido recientemente en Castilla y León. En dicho territorio, el Presidente de la Comunidad decidió adelantar el confinamiento domiciliario a las ocho de la tarde ${ }^{21}$, horario que estaba fuera de las franjas horarias establecidas en el art. 5 del RD 926/2020 y que contrastaba con las medidas, más laxas, adoptadas por otras Comunidades Autónomas con mayor número de infectados y de fallecidos. El Acuerdo del ejecutivo regional fue suspendido por el Tribunal Supremo ${ }^{22}$. Este último estimó que la medida suponía, in ictu oculi, una limitación de un derecho fundamental que no sólo iba más allá de lo permitido por la norma que declara el estado de alarma, sino que, además, resulta dictado por un órgano incompetente prima facie.

\footnotetext{
${ }^{18}$ Por ejemplo, "Francia impone el toque de queda en París y otras ocho ciudades desde el sábado para frenar la segunda ola" RTVE noticias, Disponible en: https://www.rtve.es/noticias/20201014/francia-estado-urgencia-sanitaria-desde-medianocheviernes/2045246.shtml

${ }^{19}$ Ver nota 5.

20 "Navidad en España: estas son las medidas y restricciones en las comunidades por el coronavirus", El País, 4 de enero 2020. Disponible en: https://elpais.com/sociedad/2020-1229/navidad-en-espana-estas-son-las-medidas-y-restricciones-en-las-comunidades-por-elcoronavirus.html

${ }^{21}$ Acuerdo 2/2021, de 15 de enero.

${ }^{22}$ ATS 16 febrero 2021.
} 
No parece que el establecimiento de pautas comunes pueda considerarse una interferencia centralista que limite la autonomía. Como antes se ha explicado, son aprobadas por un órgano en el que están presentes todas las Comunidades Autónomas y por mayoría, cuando no por consenso. Y, sin embargo, son imprescindibles para hacer frente a la enfermedad. Recordemos que, según el estudio de The Lancet citado al principio de estas páginas, la adopción de criterios claros y generales es la primera medida a adoptar para evitar la expansión del virus y conseguir la confianza de los ciudadanos

Hay que tener en cuenta, además, que la enfermedad no conoce límites territoriales. Explica que, por ejemplo, la Unión Europea haya actuado en ámbitos en los que sólo tiene competencias de apoyo a los Estados miembros, como son la salud pública, el empleo y las políticas fiscales y socioeconómicas. De manera distinta, en nuestro país, se ha producido una heterogeneidad que, en algunos casos, parece poco justificada. Lo cierto es que algunas Comunidades Autónomas han adoptado medidas mucho más restrictivas que otras cuando el avance de la enfermedad en las primeras era inferior al que experimentaban las segundas.

Cabe, pues, plantearse si esa diversidad no obedece más a razones de índole política que a la necesidad de luchar contra la pandemia. Es evidente que la autonomía reconocida constitucionalmente consiste, precisamente, en la posibilidad de llevar a cabo políticas distintas. Ahora bien, la autonomía, como cualquier otro poder en un Estado social y democrático de Derecho, no se justifica en sí misma, sino que es instrumental, porque debe estar al servicio de las personas.

La situación que se ha creado en nuestro país no beneficia a la legitimidad del poder. Por el contrario, puede generar una fuerte desconfianza ante los ciudadanos y perjudicar el cumplimiento de unas normas restrictivas cuya fundamentación resulta, a veces, difícil de entender. En momentos tan críticos como los actuales, cuando los derechos fundamentales están sufriendo severas restricciones, los ciudadanos y los agentes económicos esperan, entre otras cosas, que el poder actúe conforme a criterios claros y previsibles, de manera que la seguridad jurídica quede garantizada. 


\section{UN TEMA PENDIENTE: LAS GARANTÍAS DEL SISTEMA AUTONÓMICO}

En páginas anteriores, se ha analizado cómo los problemas jurídicos más graves que la lucha de la pandemia ha generado sobre nuestro modelo territorial no dependen tanto de la distribución de competencias como de la manera en que estas se están ejerciendo. Queda una última tarea por realizar, que consiste en aclarar cuál habría sido el mejor camino para evitar alguna de las dificultades arriba mencionadas. De lo expuesto anteriormente, cabe intuir que quizás las cosas habrían sido distintas si hubieran funcionado mejor las garantías que acompañan a cualquier sistema federal. Esta afirmación no está dirigida a descalificar lo que se ha hecho, sino que puede tener sentido con respecto al futuro. La lucha contra la pandemia no ha concluido y la gestión pública puede mejorarse si se confía más en dichos mecanismos de aseguramiento.

\section{Las garantías políticas}

Las primeras garantías que deberían mejorarse son de naturaleza política. Antes que nada, sería imprescindible reforzar la colaboración y, sobre todo, la coordinación entre Estado y Comunidades Autónomas, ya que dichas técnicas son esenciales para el buen funcionamiento de cualquier sistema federal.

En nuestro país, la actuación de un órgano clave, como es el Consejo Interterritorial, ha estado rodeada de obstáculos. Recordemos que la Sala de lo Contencioso-Administrativo del Tribunal Superior de Justicia de Madrid en el Auto 128/2020, del que ya se ha hablado, impidió que dicha Comunidad Autónoma aplicara el acuerdo adoptado por el Consejo Interterritorial el 30 de septiembre, texto que contenía medidas decisivas para luchar contra la enfermedad en ese momento ${ }^{23}$.

Pero los obstáculos al funcionamiento del Consejo Interterritorial no han sido sólo externos, sino también internos. En efecto, dicho órgano no siempre ha actuado de manera oportuna y con resultados satisfactorios. El establecimiento

\footnotetext{
${ }^{23}$ Ver nota 4. La fundamentación jurídica del Auto no resultaba demasiado convincente. Entre otros argumentos, se reprochaba a la Orden de la Consejería de Sanidad de la Comunidad Autónoma que aplicaba el acuerdo un vicio (la falta de habilitación legal) que, de existir, afectaba realmente a otra disposición que no era objeto del proceso, esto es, la Orden comunicada del Ministro de Sanidad de 30 de septiembre de 2020, cuyo conocimiento, además, habría debido corresponder al Tribunal Supremo.
} 
de criterios comunes, a los que antes se ha hecho referencia ${ }^{24}$, tardó casi ocho meses y, cuando se adoptaron, no tuvieron carácter ejecutivo. Se ha producido, pues, un defecto similar al que generalmente se imputa al funcionamiento del Consejo en la Unión Europea, esto es, que la necesidad de tejer consensos retrasa, cuando no impide, una toma eficaz de decisiones.

El modelo de cogobernanza que se ha implantado en nuestro España se inspira, según algunos responsables políticos, en el sistema federal alemán ${ }^{25}$. Las elevadas cifras de afectados y fallecidos que ha producido la tercera oleada de la covid en ese país son suficientes para poner en duda la bondad del modelo, que también es objeto de acerbas críticas internas ${ }^{26}$. Aun así, cabe afirmar que la estrategia que se ha seguido en España es muy distinta a la alemana. En la República Federal, hay una Ley nacional para la protección contra las infecciones, que refuerza el poder de la Federación ${ }^{27}$. La primera autoridad científica del país en la lucha contra la pandemia no es un grupo de expertos designados por el Ejecutivo sino el Instituto Robert Koch, que recoge datos y aconseja estándares médicos y sociales que son ampliamente respetados, no sólo por la Federación sino también por los Estados miembros ${ }^{28}$. Por último, la coordinación está liderada por la Canciller y por los Presidentes de los Länder. Dicha acción ha conseguido que, en los momentos más difíciles de la pandemia, la respuesta de los distintos territorios fuera uniforme $^{29}$. Y, sobre todo, el sistema de partidos políticos, que es una de las

\footnotetext{
${ }^{24}$ Ver nota 5.

${ }^{25}$ Ver, por ejemplo, "Feijóo apuesta por un modelo de "cogestión" de los fondos europeos contra la COVID similar al de los 'lander' alemanes" El Confidencial digital, Disponible en: https://www.elconfidencialdigital.com/articulo/ultima-hora/feijoo-apuesta-modelo-cogestionfondos-europeos-covid-similar-lander-alemanes/20200731165125152170.html

${ }^{26}$ Carbajosa, A, "La ley alemana de restricciones por la pandemia se topa con protestas en la calle y en la oposición", El País, 18 noviembre 2020. Disponible en: https://elpais.com/internacional/2020-11-18/la-ley-alemana-de-restricciones-por-la-pandemiase-topa-con-protestas-en-la-calle-y-en-la-oposicion.html

${ }^{27}$ Kölling, M., "Las instituciones democráticas y los derechos fundamentales en tiempos de Covid-19 en Alemania", en Biglino Campos, P.; Durán Alba, F. Los efectos horizontales de la COVID sobre el sistema constitucional, Fundación Manuel Giménez Abad, Zaragoza, 2020, p. 4. DOI: https://doi.org/10.47919/FMGA.OC20.0020

${ }^{28}$ Milbradt, G., "Federalism and the Covid-19 crisis. A perspective from Germany", Cuadernos Manuel Giménez Abad, no $19, \quad 2020, \quad$ p. 21. https://www.fundacionmgimenezabad.es/sites/default/files/Publicar/documentacion/documentos /2020/20200601_tp_milbradt_g_en_o.pdf.

${ }_{29}$ Kuhlmann, S., "Between Unity and Variety: Germany's Responses to the COVID-19 Pandemic", en Joyce, P. Maron, F., Reddy, P. S. (Ed), Good Public Governance in a Global Pandemic, p. 302. DOI: https://doi.org/10.46996/pgs.v1e1
} 
principales garantías de cualquier sistema federal, es muy diferente del nuestro.

El control político que corresponde a las Asambleas Legislativas de las Comunidades Autónomas y al Parlamento nacional podría, también, experimentar sustanciales mejoras. Antes se señalaba que el RD 926/2020 no avanza en esa dirección. Es cierto que, como principales responsables en la toma de decisiones en la lucha de la pandemia, deberían ser los Presidentes de las Comunidades Autónomas quienes respondiesen de su gestión ante las Cámaras de sus propios territorios, por lo que estaría justificado que dichas Asambleas adoptasen formas específicas de control. Pero este hecho no exime de responsabilidad al Gobierno frente a las Cortes Generales. Sobre todo, debería acentuarse el papel del Senado a la hora de llevar a cabo este tipo de fiscalización. No conviene olvidar, precisamente, que es la Cámara de representación territorial y que se trataría de valorar el uso que el Ejecutivo ha hecho de sus competencias constitucionales en materia de legislación básica y coordinación de la sanidad.

También sería necesario reforzar el principio de publicidad, que es una de las principales garantías del principio democrático. La urgencia de luchar contra la pandemia ha hecho que la función normativa de los Parlamentos quede desplazada en favor de decisiones adoptadas en el seno de los Gobiernos, órganos que no funcionan ni con luz ni con taquígrafos. De esta manera, los ciudadanos no tienen conocimiento de las razones que llevan a la adopción de determinadas decisiones, aunque estas les afecten de manera muy incisiva. Sería preciso evitar que estas medidas fueran tomadas al margen de las instituciones que representan a todos, incluidos a quienes han votado por partidos de la oposición.

En materia de transparencia habría también mucho que hacer. A pesar de la importancia que este principio adquirió a partir de la crisis económica de 2007 , no parece que las obligaciones que impone se hayan tomado muy en serio durante la gestión de la pandemia. Se han tardado varios meses en hacer públicos los nombres de quienes componen el comité de expertos que asesoró al Gobierno, a pesar de los requerimientos del Consejo de Transparencia y 
Buen Gobierno ${ }^{30}$. Las actas de las reuniones celebradas por el Consejo Interterritorial de salud todavía no se han hecho públicas. El argumento que se ha utilizado para justificar esta falta de información radica es que todas las reuniones de dicho órgano han sido extraordinarias, mientras que las actas deben ser aprobadas en sesiones ordinarias ${ }^{31}$. Dado el tiempo que ha transcurrido desde el inicio de la pandemia y de que su gestión empieza a ser algo cotidiano, no parece muy difícil convocar una reunión ordinaria para la aprobación de todas las actas que hay pendientes.

El limitado papel que han desempeñado las garantías políticas no es un dato exclusivo de nuestro sistema, sino que caracteriza a casi todos los federalismos de devolución. En estas formas de organización territorial, ese tipo de instrumentos tienden a reforzar la capacidad de integración de la Federación, por lo que despiertan las suspicacias de quienes son partidarios de reforzar el poder de los Estados miembros ${ }^{32}$.

\section{Las garantías jurídicas}

Ahora bien, lo que no tiene fácil explicación es que, en las circunstancias que estamos viviendo, tampoco hayan funcionado las garantías características de nuestro modelo de organización territorial, esto es, las que poseen naturaleza jurídica. La reflexión, al tratar este extremo, debe ser más general que la que se ha hecho hasta el momento. Como se analizará a continuación, la falta de garantías jurídicas de la distribución de competencias es una consecuencia de otro problema más acusado, esto es, la limitación de los controles inherentes al Estado de Derecho que se ha producido como consecuencia de la pandemia.

\footnotetext{
${ }^{30}$ La más reciente, de 30 de noviembre de 2020, instaba al Ministerio de Sanidad a que a que, en el plazo máximo de 10 días hábiles, remitiese información sobre número, nombre y apellidos de los expertos pertenecientes a la Dirección General de Salud Pública (Resolución 0584/2020, Resoluciones de noviembre de 2020 - Resoluciones de 2020 - Resoluciones de la Administración General del Estado - Resoluciones - Actividad - Consejo Transparencia y Buen Gobierno (consejodetransparencia.es). El Ministro hizo pública esa información a finales de diciembre del mismo año (https://www.consalud.es/pacientes/especial-coronavirus/covid-19nombres-comite-expertos-asesorado-gobierno_90218_102.html

${ }_{31}$ Ojeda, D., "Sanidad oculta las actas de las reuniones del Consejo Interterritorial durante la pandemia" El Confidencial, 28 octubre 2020, https://www.elconfidencial.com/espana/2020-1028/coronavirus-covid19-sanidad-actas-consejo-interterritorial-transparencia_2808139/

32 Biglino Campos, P. Federalismo de integración y de devolución: el debate sobre la competencia, Madrid, CEPC, 2007, pp .179-180.
} 
El dato que más llama la atención es que, muchos meses después del primer estado de alarma, el Tribunal Constitucional todavía no haya entrado a valorar si las restricciones a los derechos fundamentales y el ejercicio de las competencias durante la lucha contra la pandemia ha sido conforme a nuestra norma fundamental. Hasta el momento de escribir estas páginas, su intervención más sustantiva ha sido mediante el Auto 40/2020, sobre los límites al ejercicio del derecho de manifestación. $Y$ en esa ocasión el Tribunal, al inadmitir el recurso, evitó tratar con profundidad los límites que afectan al poder durante la pandemia. En efecto, el Auto literalmente afirma que:

La discusión sobre si el decreto de declaración del estado de alarma supone o no, de facto, y por derivación de la limitación de la libertad deambulatoria del artículo 19 CE, una limitación excesiva o incluso una suspensión del derecho de manifestación no puede ser abordada, ni siquiera a efectos dialécticos en este momento procesal, ni siquiera en este recurso de amparo (f. j. 4).

El Tribunal podría haber aprovechado otras ocasiones para dictar sentencia sobre otros problemas constitucionales que plantea la pandemia. Todavía sigue pendiente el recurso de inconstitucionalidad interpuesto contra el RD 926/2020 y la autorización de su prorroga por el Congreso de los Diputados. En marzo de este año el Pleno de dicho órgano acordó recabar para su conocimiento dos recursos de amparo interpuestos por organizaciones sindicales contra la prohibición de manifestaciones para conmemorar el día de la mujer en Madrid $^{33}$. Aunque el Tribunal Constitucional admite a trámite el amparo y reconoce que el asunto tiene transcendencia constitucional, deniega sin embargo las medidas de suspensión cautelarísima solicitadas por los recurrentes. Por último, el Tribunal Constitucional ha inadmitido por providencia dos recursos de amparo interpuestos contra autos de la Sala Tercera del Tribunal Supremo que inadmitían recursos contenciosos administrativos contra los dos RD de declaración de Estado de alarma ${ }^{34}$.

Este silencio es llamativo precisamente porque coincide con dicha negativa del Supremo a analizar la validez de esas normas. Al menos en dos ocasiones ${ }^{35}$,

\footnotetext{
${ }^{33}$ Providencias no recurso 1293-2021 y 1294-2021, 8 de marzo 2021.

${ }^{34}$ Tribunal Constitucional, gabinete del Presidente, oficina de prensa. Nota informativa $\mathrm{n}^{\circ}$ $120 / 2020$.

${ }^{35}$ ATS $11421 / 2020$, de 18 de noviembre de 2020 y ATS 12380/2020, de 22 de diciembre.
} 
dicho órgano ha decidido que los Reales Decretos que declaran el estado de alarma no son susceptibles de control por la jurisdicción contenciosoadministrativa ya que, por tener fuerza de ley, deben ser conocidos en exclusiva por el Tribunal Constitucional.

La razón de esta situación se remonta al ATC 7/2012 y a la STC 83/2016, dictadas en circunstancias muy distintas a las que vivimos. Se trataba, entonces, de decidir sobre el RD 1673/2010 y de su prorroga, que imponían la militarización los controladores aéreos, lo que conllevaba, entre otras consecuencias, cierta restricción de sus derechos fundamentales. En la Sentencia el Tribunal Constitucional reconoce que el estado de alarma no suspende derechos fundamentales, aunque si puede limitarlos (f. j. 8). El Tribunal también reivindicó su monopolio a la hora de controlar la constitucionalidad de las medidas. En efecto, el Tribunal atribuyó a dicho Real Decreto fuerza de ley, dado que disponía sobre la legalidad aplicable durante su vigencia. Junto a ello, consideró que la decisión de declarar el estado de alarma era expresión de una competencia constitucional del Gobierno en ejercicio de la función de dirección política que le atribuye el artículo 97 CE. La previa autorización de la prórroga por el Congreso de los Diputados sirve al Tribunal para reforzar su argumentación acerca de la fuerza de ley de la declaración del estado de alarma. Para dicho órgano, si se predica esa condición del acto de autorización parlamentaria, debe también postularse de la decisión gubernamental de prórroga (f. j. 10).

Las consecuencias de aplicar esa doctrina a la situación jurídica generada por la pandemia son, sin duda, gravosas para nuestro ordenamiento. En primer lugar, porque no puede compararse la incidencia que tuvo un estado de alarma que afectaba sólo a algunos derechos de un grupo muy concreto de ciudadanos con la que ha tenido los recientes estados de alarma, que han limitado, de manera mucho más intensa, muchos derechos fundamentales de toda la población y durante mucho más tiempo. Pero, además, la atribución de fuerza de ley a los Reales Decretos ha supuesto una clara restricción del control jurídico sobre el poder que es imprescindible en cualquier Estado de Derecho.

En definitiva, la situación en que nos encontramos aconseja a preguntarse si la jurisprudencia dictada por el Tribunal Constitucional para el caso de los 
controladores aéreos puede mantenerse inalterada hoy en día. Y, sobre esta solución, surgen serias dudas. En efecto, el Congreso de los Diputados sólo autoriza la prórroga del estado de alarma y no su declaración. Este último acto es adoptado únicamente por el Ejecutivo que, cuando actúa, está sometido a Derecho. En segundo lugar, porque, aunque es claro que la declaración del estado de alarma es manifestación de la función de dirección política reconocida al Gobierno en el artículo $97 \mathrm{CE}$, ello no exime del control. Hace ya años que la noción de actos políticos se desterró de nuestro ordenamiento, hecho que confirma el artículo 2 de la Ley 29/1998, reguladora de la Jurisdicción Contencioso-Administrativa, que somete a control jurisdiccional los elementos reglados de los actos del Gobierno y de los Consejos de Gobierno de las Comunidades Autónomas.

La limitación del control jurídico sobre la actuación de los Ejecutivos sobre la pandemia se ha hecho más acusada si tenemos en cuenta lo dispuesto en el artículo 2.3 del RD 926/2020. Dicho precepto habilita a los Presidentes de las Comunidades Autónomas a dictar las órdenes, resoluciones y disposiciones sobre las limitaciones de derechos fundamentales previstas en la misma norma sin tramitación de procedimiento administrativo alguno. El mismo precepto declara, además, que no es de aplicación el procedimiento previsto en los artículos 8.6 y 10.8 de la Ley 29/1998 que, como se ha señalado, prevé la ratificación o autorización judicial de las medidas sanitarias que limiten o restrinjan derechos fundamentales. Con ello, se desarticula también el control que, durante el primer estado de alarma y la nueva normalidad, habían ejercido los juzgados y las Salas de lo Contencioso-Administrativo de los Tribunales Superiores de Justicia.

Muchos de los problemas que se acaban de mencionar pueden solucionarse sin necesidad de reformas de gran calado. Una actuación decidida de nuestra justicia constitucional sería suficiente para mejorar de manera muy sustantiva las garantías jurídicas de nuestro sistema, sin necesidad de renunciar, por ello, a la eficiencia que requiere la lucha contra la pandemia. 